\title{
Análisis del aprendizaje en seguridad en una asignatura experimental de Ingeniería Química: Comparación de los resultados de la evaluación con la percepción de los estudiantes
}

\author{
María Sancho $^{\mathrm{a}}$, Beatriz García-Fayos ${ }^{\mathrm{a}}$ y José Miguel Arnal ${ }^{\mathrm{a}}$
}

${ }^{a}$ Dpto. Ingeniería Química y Nuclear, Universitat Politècnica València, Camino Vera s/n, 46022

Valencia, e-mails: msanchof@iqn.upv.es, beagarfa@iqn.upv.es, jarnala@iqn.upv.es

\begin{abstract}
Safety training is essential to strengthen a real culture of risk prevention which results in better work practices. In the field of Chemical Engineering, such training is even more important, because of the risks associated with handling of chemicals. However, the integration of risks' prevention in the new curricula of Chemical Engineering degree is very variable, and in many cases insufficient because there is not any specific subject area or compulsory courses related to this topic. Therefore, the formation of students on safety should be performed as a cross-cutting issue across the curriculum. With such aim, the Department of Chemical and Nuclear Engineering of the "Universitat Politècnica València" raised an educational innovation project to incorporate the safety training through three experimental subjects in Chemical Engineering degree. After the implementation of the project, the learning of the students in safety was tracked. This work corresponds to the assessment of safety learning at the end of the $2^{\text {nd }}$ experimental subject involved in the Project. To do that, the results achieved by the students in the academic work and at the evaluation exams are compared. These results are then compared with the perception of students about their own learning level, which was determined by means of surveys at the end of the course.
\end{abstract}

Keywords: safety, learning, tracking, Chemical Engineering

\footnotetext{
Resumen

La formación en Seguridad es esencial para consolidar una auténtica cultura de la prevención que repercuta en el ámbito laboral. En Ingeniería Química, dicha formación es aún más importante por los riesgos asociados a las sustancias químicas. Sin embargo, la formación en seguridad en los planes de estudio de Ingeniería Química es muy variable e insuficiente, pues no
} 
Análisis del aprendizaje en seguridad en una asignatura experimental de Ingeniería Química: Comparación de los resultados de la evaluación con la percepción de los estudiantes

existe ninguna materia específica, ni asignaturas troncales al respecto. Así, la formación en Seguridad debe hacerse transversalmente a través de otras asignaturas. Con este objetivo, se planteó un Proyecto de Innovación Educativa para incorporar la formación en Seguridad a través de tres asignaturas experimentales en Ingeniería Química. Tras su implantación, se realizó un seguimiento del aprendizaje de los alumnos en seguridad. Este trabajo corresponde al análisis del aprendizaje realizado al final de la $2^{\circ}$ asignatura experimental implicada en el Proyecto. Para ello, se analizaron los resultados alcanzados por los estudiantes en los trabajos académicos y en las pruebas de evaluación. En este trabajo, se comparan los resultados de dicho análisis con la percepción de los estudiantes de su propio grado de aprendizaje, determinada a partir de encuestas de valoración realizadas al finalizar la asignatura.

Palabras clave: seguridad, aprendizaje, seguimiento, Ingeniería Química

\section{Introducción}

El desarrollo industrial requiere de la mejora de la seguridad de las industrias para conseguir que dicho desarrollo sea sostenible. Esta situación requiere la aplicación de medidas de seguridad adicionales para reducir los accidentes que pueden ocurrir en las industrias. El ingeniero químico tiene un papel importante en la prevención y reducción de los riesgos asociados a los accidentes que pueden suceder en una planta química (Darbra, 2012). Por ello, la "cultura preventiva” debe ser una parte esencial de la mentalidad de un ingeniero químico (Gillet, 2001).

Para crear una cultura preventiva en los estudiantes del Grado en Ingeniería Química, los planes de estudio deben incluir la formación en materia de seguridad. Solamente mediante la práctica en seguridad a través de la educación esta disciplina puede llegar a formar parte del marco conceptual de los ingenieros químicos, tanto como estudiantes como a nivel profesional; y podrán así ser conscientes del modo en que sus decisiones influyen en las condiciones de seguridad de las industrias (Behm, 2014). Aunque la formación específica sobre la seguridad de un determinado proceso es responsabilidad de la propia industria; la eficiencia de dicha formación será considerablemente mejor cuando se trabaje con ingenieros con una cultura de seguridad positiva. De acuerdo con algunas importantes instituciones internacionales relacionadas con la seguridad industrial, es esencial empezar esta formación en la universidad (Louvar, 2009). Por ello, la seguridad debería ser una disciplina troncal de todas las titulaciones de Ingeniería Química (Pitt, 2012), de forma que los futuros ingenieros puedan satisfacer las expectativas de la sociedad para alcanzar la excelencia en materia de seguridad (Hendershot, 2007).

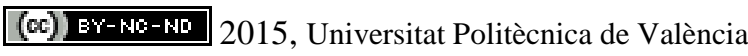

Congreso IN-RED (2015) 
Actualmente, la incorporación de la disciplina de seguridad en los grados de Ingeniería Química se ha convertido en una necesidad y una realidad, que se está implementando en un número cada vez mayor de universidades (Darbra, 2012).

Tradicionalmente, los planes de estudio del ingeniero químico tienen una parte importante de experiencia práctica en laboratorio, la cual es esencial para entender los fundamentos de la ingeniería química (Peñas, 2006). Además, los laboratorios de ingeniería química suponen un gran potencial para la formación en otras prácticas industriales como: seguridad y salud, gestión de residuos, mantenimiento, etc. (Abu-Khalaf, 2001). De hecho, en algunas universidades se ha implementado la formación en seguridad a través de asignaturas experimentales, en muchos casos usando metodologías tipo "role-playing" (Darbra, 2012; Abu-Khalaf, 2001; Graells, 2007).

En la Universitat Politècnica de València la formación en seguridad se ha integrado en el plan de estudios del Grado de Ingeniería Química a través de las tres asignaturas de "Experimentación en Ingeniería Química”. Esto se hizo en el marco de un Proyecto de Innovación y Mejora de la Docencia implementado en el curso académico 2013-14. El proyecto consistió básicamente en la definición de diferentes estrategias, materiales y actividades para incorporar el aprendizaje en seguridad a través de las sesiones experimentales de laboratorio (Arnal, 2013; Arnal, 2014).

\section{Objetivos}

Tras la implementación del proyecto de innovación, se ha realizado el seguimiento del aprendizaje en seguridad en las asignaturas objeto del proyecto. Este trabajo se refiere concretamente al análisis del aprendizaje realizado al final de la segunda asignatura experimental implicada en el Proyecto (“Experimentación en Ingeniería Química II”). Los objetivos específicos de dicho seguimiento son los siguientes:

- $\quad$ Analizar los resultados alcanzados por los estudiantes en materia de seguridad, tanto en los trabajos académicos como en las pruebas de evaluación parciales.

- $\quad$ Diseñar una herramienta para registrar la percepción de los estudiantes de su grado de aprendizaje en seguridad.

Comparar los resultados de los trabajos académicos y de las evaluaciones parciales con la percepción de los alumnos.

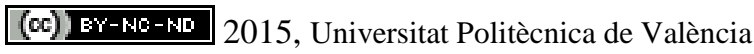

Congreso In-Red (2015) 
Análisis del aprendizaje en seguridad en una asignatura experimental de Ingeniería Química: Comparación de los resultados de la evaluación con la percepción de los estudiantes

\section{Desarrollo de la innovación}

\subsection{Seguimiento del grado de aprendizaje en seguridad}

La asignatura en la que se ha llevado a cabo el seguimiento del aprendizaje en seguridad es “Experimentación en Ingeniería Química II” (EIq2), que se imparte durante el 3er curso del Grado en Ingeniería Química, en el cuatrimestre A. En el curso académico objeto de este trabajo, la asignatura ha tenido un total de 71 estudiantes matriculados, que se dividieron en 20 grupos de 4-5 componentes.

Una de las herramientas empleadas para analizar el aprendizaje en seguridad, ha sido la tarea sobre dicha disciplina incluida en los informes de laboratorio que cada grupo de estudiantes realiza en cada sesión experimental. La tarea consiste en elaborar tablas de seguridad para las sustancias químicas empleadas en la práctica, incluyendo la información reflejada en la Tabla 1. Para completar dicha tabla, los estudiantes deben primero buscar la FDS (Ficha de Datos de Seguridad) de las sustancias en una fuente adecuada (preferiblemente del suministrador comercial) y luego localizar la siguiente información: pictogramas de peligro, frases $\mathrm{H}$ (peligro) y frases $\mathrm{P}$ (prudencia).

Tabla 1. Información requerida en la tabla de seguridad de los informes de laboratorio

\begin{tabular}{|l|l|l|l|}
\hline Sustancia química y número CAS & Pictogramas & Frase H (peligro) & Frase P (prudencia) \\
\hline & & & \\
\hline
\end{tabular}

Puesto que los estudiantes realizan varias sesiones experimentales a lo largo del cuatrimestre, se ha podido analizar el nivel de evolución en la realización de la tarea desde el informe de la primera sesión hasta el de la última . Este análisis se discutirá más adelante en el apartado de resultados.

Otra herramienta empleada para evaluar el aprendizaje en seguridad ha sido la pregunta sobre dicha materia incluida en los dos exámenes escritos realizados en el primer y segundo parcial, respectivamente. Las calificaciones obtenidas por los estudiantes en estas cuestiones se comentan en el apartado de resultados de este trabajo.

\subsection{Herramienta para registrar la percepción de los estudiantes de su grado de aprendizaje}

Para registrar la percepción de los estudiantes de su propio grado de aprendizaje se diseñó una encuesta anónima, en la que se debían valorar los distintos ítems de acuerdo a una escala de Likert de 4 niveles, como se muestra en la Tabla 2.

(c)) EY-NC-ND 2015, Universitat Politècnica de València

Congreso IN-RED (2015) 
María Sancho, Beatriz García-Fayos y José Miguel Arnal

Tabla 2. Escala de Likert empleada en la encuesta de percepción de los estudiantes

\begin{tabular}{|c|c|c|c|}
\hline 1 & 2 & 3 & 4 \\
\hline Nada & Poco & Bastante & Mucho \\
\hline
\end{tabular}

La encuesta se dividió en dos partes. En la primera, se pedía a los alumnos que valoraran sus conocimientos de seguridad en el laboratorio al principio y al final de la asignatura. Y en la segunda parte, se pedía indicar el grado de mejora del aprendizaje en los siguientes ítems más específicos:

a) Búsqueda de Fichas de Seguridad

b) Interpretación de Pictogramas

c) Identificación de Frases $\mathrm{H}$ y P

d) Uso de Equipos de Protección y ropa de trabajo

e) Conocimiento de legislación sobre Seguridad

f) Manipulación de productos químicos

En el siguiente apartado se analizan los resultados de la encuesta, y se comparan con los resultados en los trabajos académicos y en las pruebas de evaluación.

\section{Desarrollo de la innovación}

\subsection{Análisis del grado de aprendizaje en seguridad}

En primer lugar, se analizan los resultados alcanzados por los estudiantes en la tarea de elaboración de las tablas de seguridad de los informes de laboratorio. Para ello, los resultados se han dividido en los siguientes tres ítems:

1. Pictogramas. Los alumnos identificar los pictogramas y buscar su significado de acuerdo con la legislación vigente. La tarea se ha clasificado como correcta si se identificaban todos y cada uno de los pictogramas, y además el significado de todos era correcto.

2. Frases H\&P. Se debían identificar las frases de peligro $(\mathrm{H})$ y prudencia $(\mathrm{P})$ mostradas en la FDS. El ítem se ha considerado correcto cuando se han identificado correctamente todas las frases que figuran en la FDS y se han situado en el lugar correcto de la tabla.

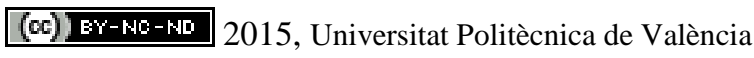

Congreso In-Red (2015) 
Análisis del aprendizaje en seguridad en una asignatura experimental de Ingeniería Química: Comparación de los resultados de la evaluación con la percepción de los estudiantes

3. Búsqueda de la FDS. La Ficha de Datos de Seguridad debía buscarse en la página web de un suministrador oficial de sustancias químicas o de un organismo oficial, como puede ser el Ministerio competente al respecto. Sólo si se buscaba en una de estas páginas web el ítem se ha considerado correcto.

Siguiendo estas directrices, se ha analizado si el ítem se realizó correctamente, de acuerdo a la tarea definida, para los 20 grupos de alumnos que trabajaron en la asignatura. Estos resultados se representan en la Fig. 1 en términos de porcentaje, donde se comparan los informes realizados en la primera y en la última práctica de laboratorio.

En la Fig. 1, se puede observar claramente que todos los grupos han mejorado significativamente en la realización de las tablas de seguridad. En los informes iniciales, más del 50\% de los grupos cometieron errores en todos los ítems analizados: algunos eran incompletos o incluso incorrectos, principalmente debido al hecho de haber buscado la FDS en una fuente inadecuada. Sin embargo, después de practicar la tarea de realización de la tabla en los siguientes informes, todos los grupos aprendieron a buscar correctamente las FDS, y todos ellos identificaron correctamente los pictogramas. En lo que respecta a las frases $H \& P$, solo un grupo cometió un pequeño error en este ítem debido a la omisión de algunas frases P. Por lo tanto, se puede afirmar que la práctica durante el cuatrimestre en la realización de la tabla de seguridad resultó en una mejora considerable del nivel de desarrollo de la tarea.

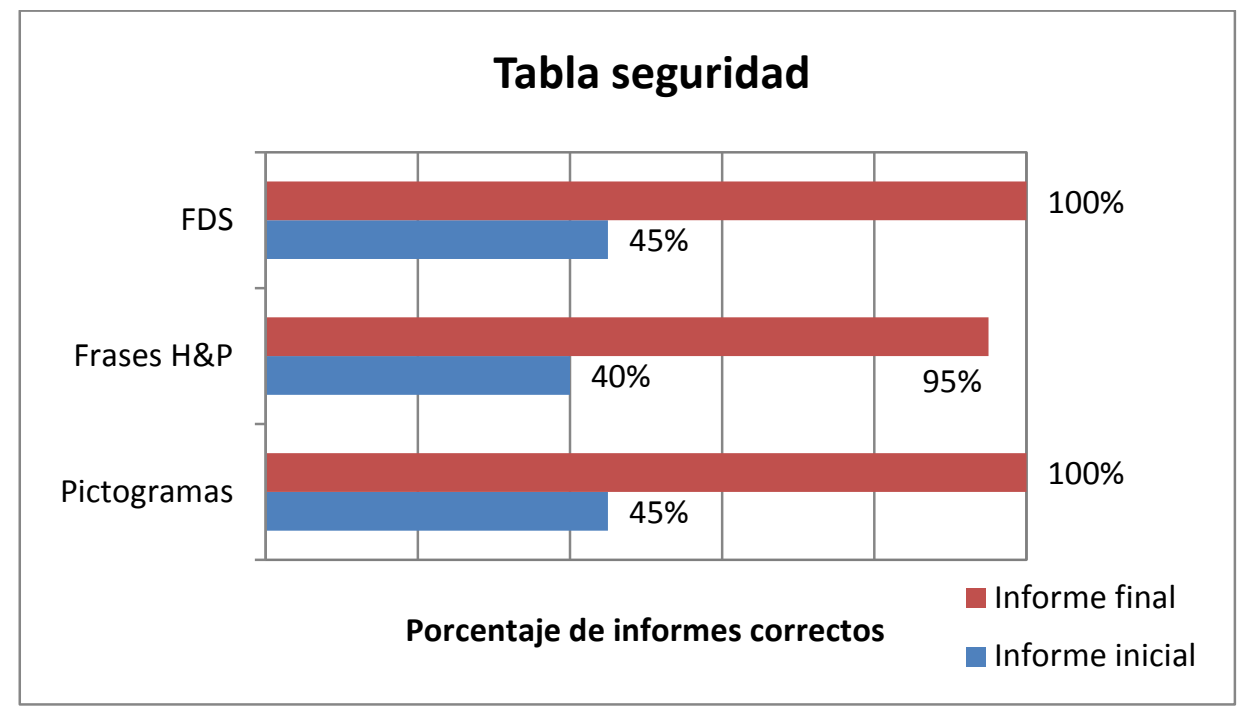

Fig. 1. Análisis de los ítems de la tabla de seguridad de los informes de laboratorio 
El aprendizaje en seguridad se analizó también a través de las calificaciones obtenidas en los dos exámenes parciales. La Fig. 2 muestra los resultados de los estudiantes en la cuestión de seguridad del primer parcial, comparada con los resultados de la cuestión de seguridad del segundo parcial. En ambos parciales, la cuestión sobre seguridad fue calificada sobre 2 puntos, y las calificaciones se han agrupado en cuatro rangos para analizar los resultados: inferiores a 0,5 puntos, entre 0,75 y 1 punto, entre 1,25 y 1,5 puntos, y superiores a 1,5 puntos.

Como se puede observar en la Fig. 2, la mayoría de los estudiantes (alrededor del 90\%) obtuvieron calificaciones por encima de 1 punto en ambos parciales. En el primer parcial, la mayor parte de las calificaciones (61,4\%) se situó en el rango entre 1,25 y 1,5 puntos. Sin embargo, la distribución de calificaciones del segundo parcial se observa claramente desplazada hacia mejores calificaciones, ya que un 52,9\% de los estudiantes obtuvo puntuaciones superiores a 1,5. Esto se puede asociar al trabajo realizado en la tabla de seguridad de los informes a lo largo del cuatrimestre, al hecho de haber practicado en más sesiones experimentales, y al feedback proporcionado a los estudiantes tras el primer parcial. Por lo tanto, se puede afirmar que el trabajo de seguridad llevado a cabo en las sesiones de laboratorio ha producido resultados muy satisfactorios en ambos parciales, y que se han obtenido mayores calificaciones al final del cuatrimestre como resultado del trabajo progresivo.

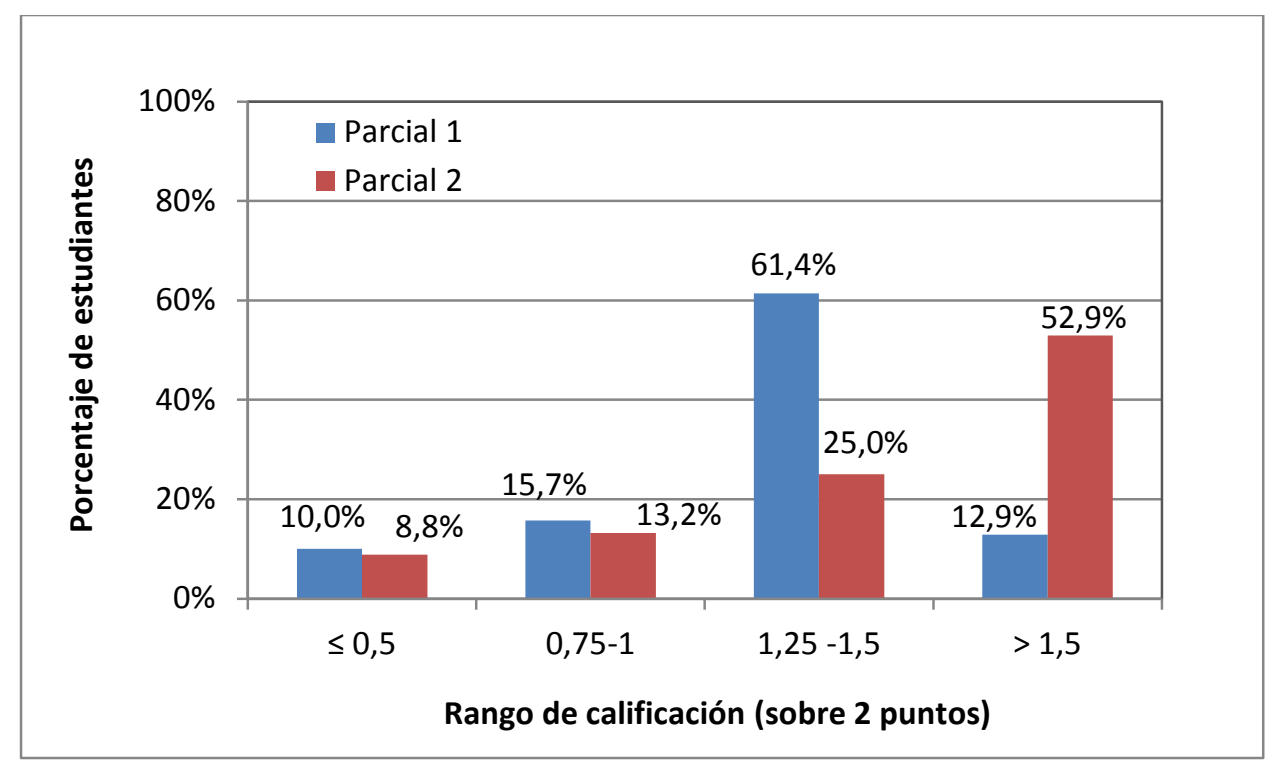

Fig. 2. Calificaciones (sobre 2 puntos) de la cuestión de seguridad en los exámenes parciales 
Análisis del aprendizaje en seguridad en una asignatura experimental de Ingeniería Química: Comparación de los resultados de la evaluación con la percepción de los estudiantes

\subsection{Análisis de la percepción de los estudiantes de su grado de aprendizaje}

En la Fig. 3 se muestran los resultados de la primera parte de la encuesta, como porcentaje de estudiantes que indicaron cada nivel de la escala para valorar sus conocimientos en seguridad al principio y al final de la asignatura.

Como se puede observar, un elevado porcentaje de estudiantes $(77,6 \%)$ valoró como "poco" su conocimiento en materia de seguridad al inicio de la asignatura, y un porcentaje entorno al $10 \%$ consideró que sus conocimientos eran nulos al principio. Observando las valoraciones al final de la asignatura, se puede afirmar que los estudiantes han percibido en conjunto una mejora considerable de su aprendizaje en seguridad, ya que el 97\% opina que su conocimiento ha crecido "bastante" o "mucho" (de acuerdo a la escala de valoración empleada). El porcentaje más significativo corresponde a la valoración de "bastante” con un $59,7 \%$.

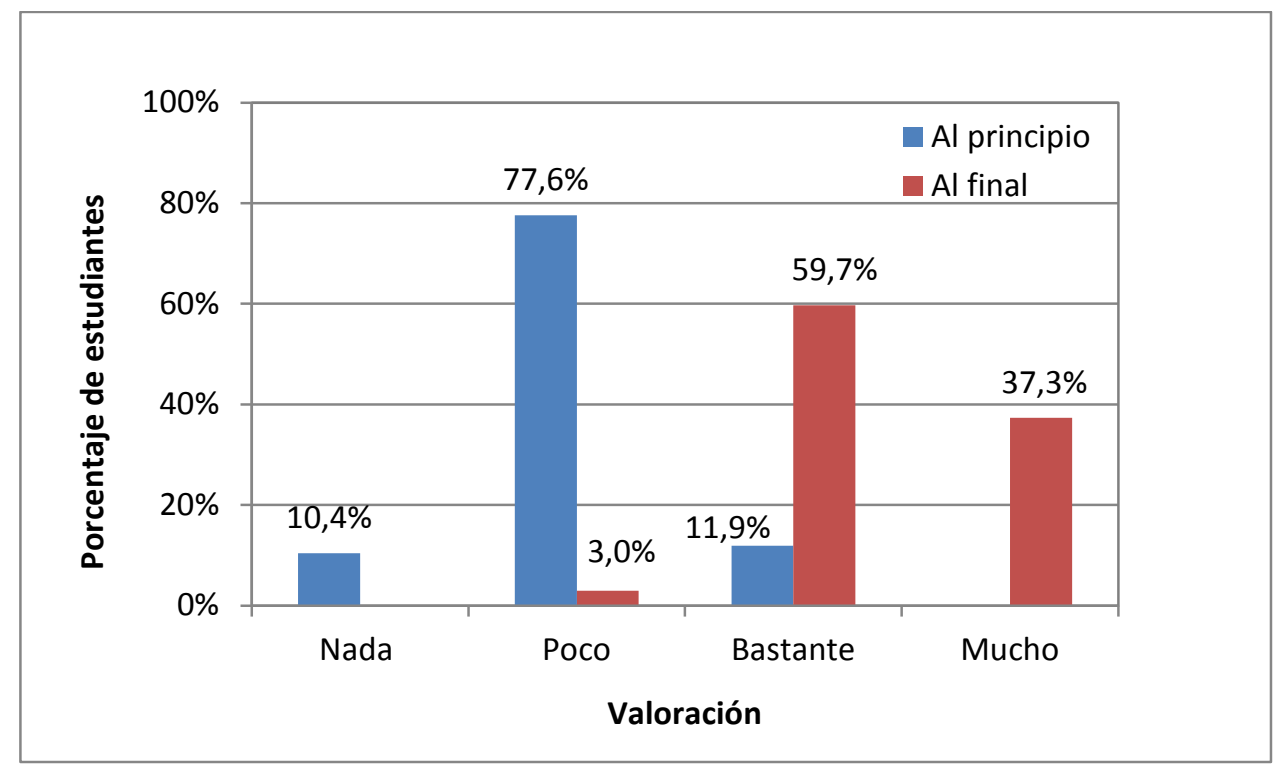

Fig. 3. Percepción de los estudiantes de la evolución de sus conocimientos en seguridad en la asignatura

Para poder analizar el grado de mejora percibido por los alumnos, en la Fig. 4 se presentan los resultados de esta primera parte de la encuesta pero desde el punto de vista de la evolución señalada por cada estudiante. Así, se observa que la mayor parte de los encuestados (49,3\%) señalaron una evolución de 2 ("poco”) a 3 ("bastante”), seguidos por 
un porcentaje del 28,4\% que consideraron que su evolución había sido de 2 (“poco”) a 4 (“mucho”). Agrupando las evoluciones que suponen un nivel de mejora (1-2, 2-3 y 3-4), éstas suman un total del 61,2\%; mientras que las evoluciones que implican dos niveles de mejora suman un total del 35,8\%.

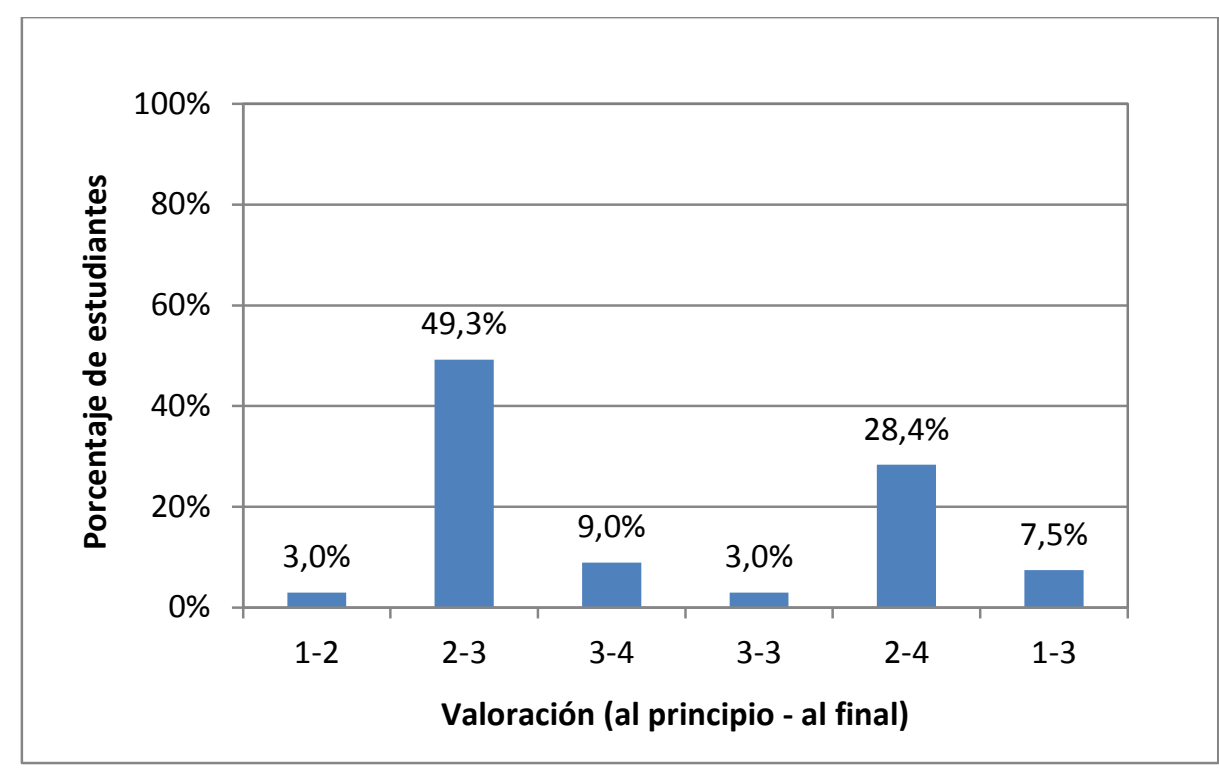

Fig. 4. Nivel de mejora en conocimientos de seguridad percibido por los estudiantes en la asignatura

Por otra parte, en la Fig. 5 se presentan las valoraciones de los estudiantes del grado de mejora en los ítems correspondientes a la segunda parte de la encuesta. Observando en conjunto todos los ítems, se aprecia una valoración muy positiva del grado de aprendizaje, ya que casi todos ellos son percibidos por los estudiantes con escalas de mejora de “bastante” o "mucho”. Concretamente, para cada ítem se puede establecer lo siguiente:

a) Búsqueda de FDS. La mayor parte de las encuestas sitúan la percepción del grado de mejora repartida en porcentajes similares entre "bastante” (44,8\%) y “mucho" (41,8\%).

b) Pictogramas. En este caso, hay un porcentaje más significativo de valoraciones del grado de aprendizaje como "bastante" (61,2\%).

c) Frases H\&P. Este ítem es el que cuenta con un porcentaje mayor en una de las valoraciones, concretamente el 64,2\% de los alumnos opinó que su grado de mejora había sido "bastante" en este apartado.

(cc) EY-NC-ND 2015, Universitat Politècnica de València

Congreso In-Red (2015) 
Análisis del aprendizaje en seguridad en una asignatura experimental de Ingeniería Química: Comparación de los resultados de la evaluación con la percepción de los estudiantes

d) EPIs. En este caso, los resultados de las encuestas sitúan la percepción del grado de mejora de este ítem repartida básicamente entre las valoraciones de "bastante” (41,8\%) y “mucho" (50,7\%).

e) Legislación. Este es el ítem que ha sido señalado como el de menor grado de mejora, con un porcentaje mayor $(47,8 \%)$ en la valoración "poco" de la escala. Este resultado es coherente con la formación recibida por los estudiantes en el ítem, ya que es el que menos se ha trabajado en la asignatura. Esto que confirma que la encuesta ha sido respondida adecuadamente por los estudiantes.

f) Manipulación. La mayor parte de las encuestas sitúan la percepción del grado de mejora de este apartado en la valoración de "bastante”, con un porcentaje del 50,7\%.

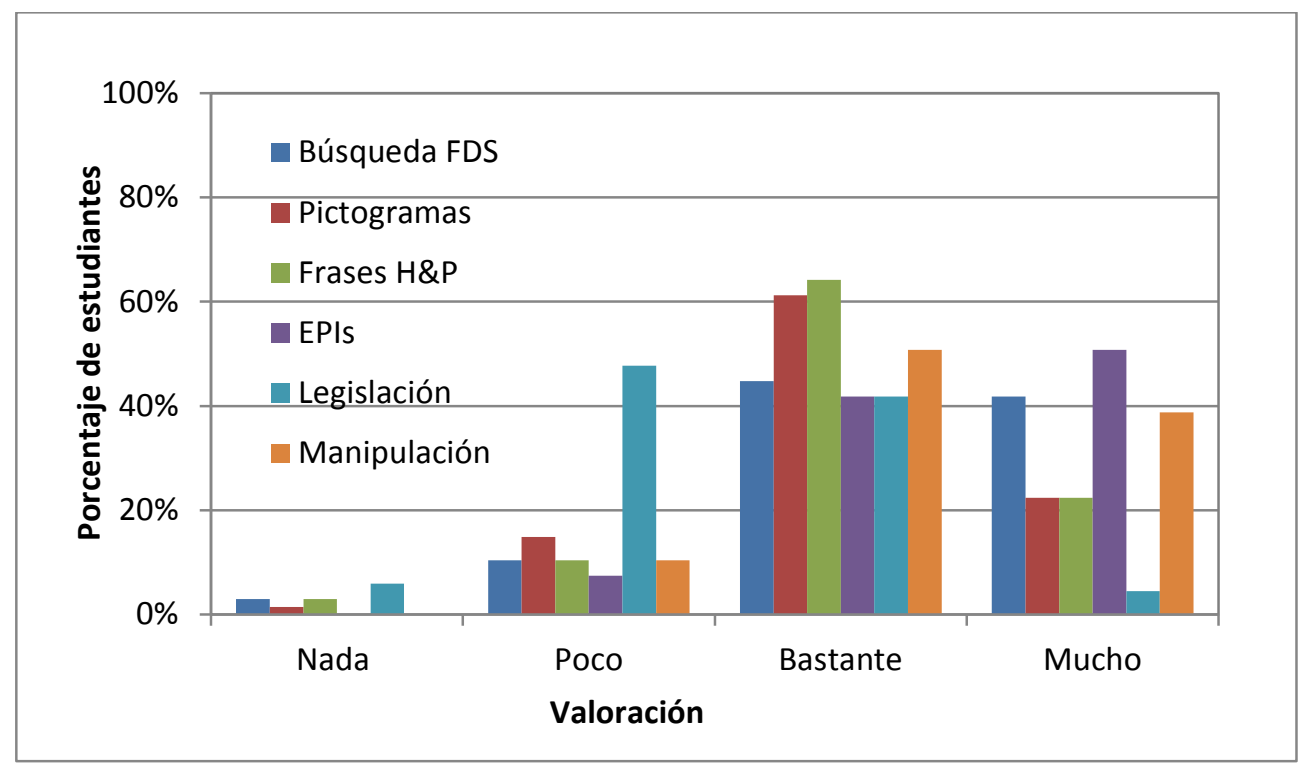

Fig. 5. Percepción de los estudiantes del grado de mejora en apartados de seguridad específicos

\subsection{Comparación de los resultados de aprendizaje con la percepción de los estudiantes}

En la Fig. 6 se muestran los resultados del grado de mejora de los alumnos en la asignatura, determinados del siguiente modo: 
- $\quad$ En el caso de las calificaciones de los exámenes, se han comparado las notas sobre dos puntos en la cuestión sobre seguridad del primer y del segundo parcial, determinando para cada estudiante si su calificación empeoró, se mantuvo dentro del mismo rango o aumentó dentro de los rangos definidos en uno o más niveles.

En el caso de los resultados de la encuesta, se ha agrupado la evolución señalada por cada estudiante de acuerdo a los cuatro casos señalados en el caso de las calificaciones. Cabe señalar que en el momento de responder a la encuesta los estudiantes todavía no habían realizado el segundo parcial de la asignatura.

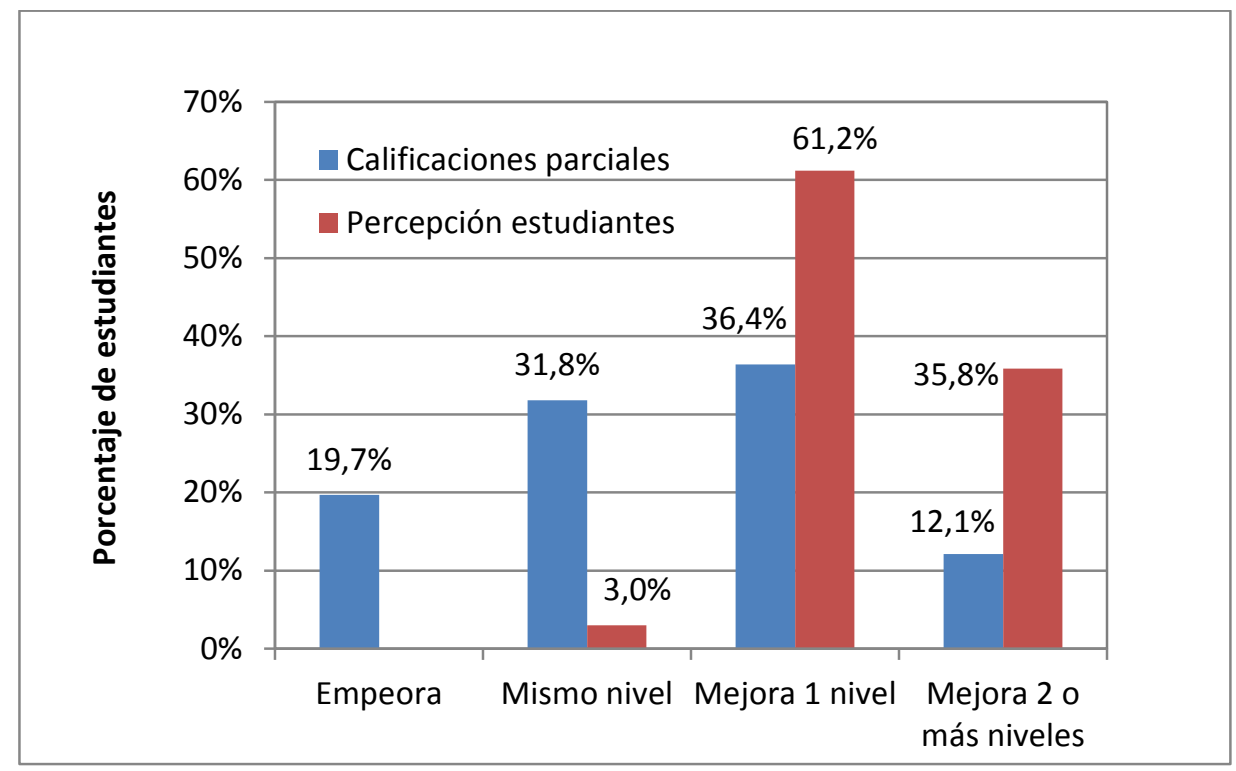

Fig. 6. Comparación del nivel de mejora en las calificaciones con la percepción de los estudiantes

En la Fig. 6 se puede observar que la percepción de los estudiantes de su grado de aprendizaje global en la asignatura resulta bastante optimista en relación con los resultados alcanzados en los parciales. Sólo un 3\% de los alumnos consideraron que su aprendizaje no había mejorado, repartiendo el restante $97 \%$ en una mejora entre 1 y 2 niveles. Sin embargo, los resultados de la cuestión de seguridad de los exámenes parciales muestran que la mejora en las calificaciones no ha sido tan relevante, pues el porcentaje de calificaciones mejoradas es algo inferior al 50\% (48,5\%). Cabe mencionar, de todas formas, que un porcentaje entorno al $11 \%$ de las calificaciones que mantuvieron el mismo nivel ya partían de una elevada puntuación (mayor de 1,5 puntos frente a un total de 2). Por otra parte, en la encuesta no se ha registrado ningún alumno que indicara un empeoramiento de su

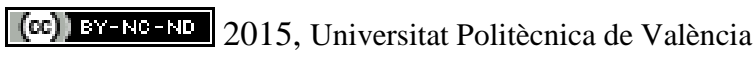

Congreso In-Red (2015) 
Análisis del aprendizaje en seguridad en una asignatura experimental de Ingeniería Química: Comparación de los resultados de la evaluación con la percepción de los estudiantes

aprendizaje, lo cual denota que los estudiantes se han podido basar más en el trabajo de laboratorio que en los exámenes para valorar su grado de mejora. Además, habría que considerar que la cuestión de seguridad del examen no recoge exactamente todo lo que abarca el aprendizaje de seguridad de los alumnos, por lo que de cara al futuro habría que desarrollar alguna herramienta de evaluación que recogiera de forma más completa la evidencia del grado de aprendizaje.

De hecho, si se compara la evolución de los resultados de las tablas de seguridad de los informes (Figura 2) con la percepción de los estudiantes en los ítems que se trabajan en dicha tabla (FDS, pictogramas y frases H\&P), reflejados en la Figura 6, se observa una mayor coherencia entre la mejora observada y la percepción de los estudiantes. Por lo tanto, podría decirse que las herramientas para analizar la evolución del aprendizaje deben contemplar necesariamente las evidencias del trabajo realizado a lo largo de las sesiones experimentales, además de las calificaciones en los exámenes.

\section{Conclusiones}

En este trabajo se ha analizado el grado de aprendizaje en seguridad en una asignatura experimental, tras la implantación de un Proyecto de Innovación y Mejora de la Docencia. El análisis llevado a cabo permite establecer las siguientes conclusiones:

- El trabajo continuo y progresivo en sesiones experimentales mejora considerablemente el grado de aprendizaje en seguridad, reflejado tanto en los trabajos académicos como en el desplazamiento de las calificaciones en la materia hacia rangos de mayor puntuación.

- Las calificaciones en los exámenes no pueden ser la única evidencia del grado de aprendizaje de los estudiantes, ya que hay determinados aspectos del aprendizaje que los alumnos valoran como adquiridos positivamente que no siempre se contemplan en el examen escrito.

- Los resultados de aprendizaje de los trabajos académicos, realizados de forma progresiva y con el feedback correspondiente, pueden ser una buena evidencia de la evolución del aprendizaje de los estudiantes.

\section{Referencias}

ABU-KHALAF, A.M. (2001). "Introducing safety in the chemical engineering laboratory course”. Chemical Health \& Safety, January/February, pp. 8-11. 
ARNAL, J.M., SANCHO, M., GARCÍA-FAYOS, B. (2014). “Adaptación de la docencia impartida en una asignatura experimental del plan extinguido de Ingeniero Químico a la nueva estructura de asignaturas del Grado de Ingeniería Química”. Jornadas INRED-14.

ARNAL, J.M., SANCHO, M., GARCÍA-FAYOS, B. (2013). "Integration of industrial hygiene and safety in experimental subjects of chemical engineering degree”. Proceedings of International Technology, Education and Development Conference-INTED13.

BEHM, M.; CULVENOR, J.; DIXON, G. (2014). "Development of safe design thinking among engineering students”. Safety Science, 63, pp. 1-7.

DARBRA, R.M.; CASAL, J.; PASTOR, E.; VÍLCHEZ, J.A.; ARNALDOS, J.; PLANAS, E. (2012). "Risk analysis active learning through the investigation of real cases". Process Safety and Environmental Protection, 90, pp. 430-435.

GILLET, J.E. (2001). “Chemical engineering education in the next century”. Chem. Eng. Technol. 24, pp. 561-570.

GRAELLS SOBRÉ, M.; PÉREZ-MOYA, M. (2007). “Projecte PEEEQ _ Planificació estratégica de les assignatures d’Experimentació en Enginyeria Química de l'Escola Universitària d’Enginyeria Tècnica Industrial de Barcelona”. 10è Premi a la Qualitat en la Docència Universitària, Consell Social Universitat Poltècnica de Catalunya

HENDERSHOT, D.C.; SMADES, W. (2007). "Safety culture begins in the classroom”. Process Safety Progress, 26 (2), pp. 83-84.

LOUVAR, J.F. (2009). “Safety and Chemical Engineering education _ History and results”. Process Safety Progress, 28 (2), pp. 131-134.

PEÑAS, F.J.; BARONA, A.; ELÍAS, A.; OLAZAR, M. (2006). "Implementation of industrial health and safety in chemical engineering teaching laboratories”. J. Chemical Health \& Safety, March/April, pp. 19-23.

PITT, M.J. (2012). “Teaching safety in chemical engineering: what, how and who?”. Chem. Eng. Technol. 35 (8), pp. 1341-1345. 